\title{
Point Measurements of Fermi Velocities by a Time-of-Flight Method
}

\author{
Falk, David S.; Henningsen, J. O.; Skriver, Hans Lomholt; Christensen, N. Egede
}

Published in:

Physical Review B Condensed Matter

Link to article, DOI:

10.1103/PhysRevB.6.377

Publication date:

1972

Document Version

Publisher's PDF, also known as Version of record

Link back to DTU Orbit

Citation (APA):

Falk, D. S., Henningsen, J. O., Skriver, H. L., \& Christensen, N. E. (1972). Point Measurements of Fermi Velocities by a Time-of-Flight Method. Physical Review B Condensed Matter, 6(2), 377-391.

https://doi.org/10.1103/PhysRevB.6.377

\section{General rights}

Copyright and moral rights for the publications made accessible in the public portal are retained by the authors and/or other copyright owners and it is a condition of accessing publications that users recognise and abide by the legal requirements associated with these rights.

- Users may download and print one copy of any publication from the public portal for the purpose of private study or research.

- You may not further distribute the material or use it for any profit-making activity or commercial gain

- You may freely distribute the URL identifying the publication in the public portal 


\title{
Point Measurements of Fermi Velocities by a Time-of-Flight Method*
}

\author{
David S. Falk \\ Department of Physics and Astronomy, University of Maryland, College Park, Maryland 20742 \\ and \\ J. O. Henningsen \\ Physics Laboratory I, H. c. Ørsted Institute, Universitetsparken 5, Copenhagen, Denmark \\ and \\ H. L. Skriver and N. E. Christensen \\ Physics Laboratory I, Technical University of Denmark, 2800 Lyngby, Denmark \\ (Received 20 January 1972)
}

\begin{abstract}
The present paper describes in detail a new method of obtaining information about the Fermi velocity of electrons in metals, point by point, along certain contours on the Fermi surface. It is based on transmission of microwaves through thin metal slabs in the presence of a static magnetic field applied parallel to the surface. The electrons carry the signal across the slab and arrive at the second surface with a phase delay which is measured relative to a reference signal; the velocities are derived by analyzing the magnetic field dependence of the phase delay. For silver we have in this way obtained one component of the velocity along half the circumference of the centrally symmetric orbit for $\vec{B} \|[100]$. The results are in agreement with current models for the Fermi surface. For $\vec{B} \|[011]$, the electrons involved are not moving in a symmetry plane of the Fermi surface. In such cases one cannot immediately derive the velocity components, but the method can still be used to provide a comparison of different Fermi surface models. Such a comparison has been made of an augmentedplane-wave model (Christensen) and a Fourier model (Halse), both yielding the experimentally determined areas and cyclotron masses for symmetry orbits of the Fermi surface, but differing slightly at general points. The comparison favors the Fourier model.
\end{abstract}

\section{INTRODUCTION}

Most experimental information involving the Fermi velocity of electrons in metals is obtained in the form of averages over closed electron orbits, and more or less complicated inversion schemes must be devised in order to extract from such data the velocities at isolated points on the Fermi surface. ${ }^{1}$ One notable exception concerns the magnetic surface states, ${ }^{2}$ a study of which allows a point-by-point mapping of the Fermi velocity along certain contours. However, even in this case, the analysis requires a thorough knowledge of the geometry of the Fermi surface in the vicinity of these points.

In this paper we discuss a different technique which in many cases makes it possible to obtain velocity information without presupposing more than a qualitative understanding of the Fermi-surface shape. The method is based on the transmission of microwave power through a metal slab in the presence of a magnetic field applied parallel to the surface. This is the configuration appropriate to the radio-frequency size effect. We shall be concerned with the range of magnetic fields below the first-order size-effect line, where all electrons collide with at least one metal surface. The electrons are accelerated by the electric field while they are in the skin layer, and they subse- quently follow a curved trajectory in the magnetic field until, after a certain time of flight, they collide with the second surface. Here they give rise to a transmitted signal whose phase is determined by the time of flight, i. e., by the reciprocal of the electron velocity, integrated over the trajectory. The idea is now to measure this time of flight by using the microwave period as a time unit. This is done by letting the transmitted signal interfere with a leak signal, whose phase is magnetic field independent, and observing the beat pattern. For accurate measurements we require a time of flight of many time units, and since the time of flight is a decent fraction of a cyclotron period, we must have the microwave frequency large compared to the cyclotron frequency. We thus find ourselves working at frequencies typical of cyclotron resonance rather than of size-effect experiments. The time of flight in itself contains velocity information in the form of an average over the trajectory. However, a differential change in magnetic field leads to a corresponding change in the length of the trajectory. The difference in time of flight at the two magnetic fields is then directly related to the velocity at the end point of the trajectory. This point can be moved around on the Fermi surface by sweeping the magnetic field, and by applying a straightforward analysis, whose basic ingredient is a differentiation of the time of flight with re- 
spect to magnetic field, we are therefore able to perform the desired point-by-point mapping.

The present work is based on experimental evidence reported briefly in a previous publication. ${ }^{3}$ We begin in Secs. II-IV by describing in some detail the experimental technique, emphasizing the microwave aspects as well as the sample preparation. We then in Sec. $V$ review the experimental results obtained for the magnetic field along the [011] and [100] directions in the (011) plane of silver. The origin of the results, which are qualitatively different for the two directions, is explained in Sec. VI. Using a simple model, which serves only to elucidate the principles of the transmission process, it is shown that the observed oscillations arise from electrons that collide with either one or two metal surfaces. (From here on these are denoted type-I and type-II electrons, respectively.) We proceed in Sec. VII with the development of a more rigorous theory which will eventually enable us to extract velocities from the experimental tracings, or, alternatively, to calculate transmission spectra if the velocities are known. The first step is a derivation of a variational expression for the transmission coefficient. Next, this quantity is evaluated by using a damped exponential as a trial function for the electric field distribution. It is here necessary to consider type-I and type-II contributions separately. We do not attempt to perform a self-consistent calculation, and the results we obtain seem to justify this omission.

Since the objective of this paper is to demonstrate the applicability of a new technique, we rely heavily on augmented-plane-wave (APW) calculations of Fermi velocities, performed by Christensen. ${ }^{4}$ Using these in conjunction with our derived transmission coefficient, we calculate transmission spectra for the two orientations, and these are compared with the experimental tracings in Sec. VIII. For $\overrightarrow{\mathrm{B}} \|[100]$, where type-II electrons are dominant, the agreement is almost perfect in the entire range of magnetic fields considered. For $\vec{B} \|[011]$, we find good agreement in most of the range if we base our interpretation on type-I trajectories alone. Some minor discrepancies at low magnetic fields, as well as the discomforting absence of a type-II contribution, are discussed.

In Sec. IX we reverse the procedure. We now assume the velocities to be unknown and develop a procedure by which we can extract them from the observed spectra. It turns out that the $\vec{B} \|[100]$ case allows determination of one velocity component to an accuracy of a few percent over a $45^{\circ}$ range of the central orbit on the Fermi surface. This corresponds by symmety to one-half of the entire circumference. An experiment with the sample cut in a (001) plane would supplement this information to provide both velocity components along the entire orbit. In contrast, the $\vec{B} \|[011]$ case proves more difficult to analyze. Owing to the presence of the necks, the orbits involved are not located in a symmetry plane. Different orbits are operative at different magnetic fields, and the contour along which the velocity is measured can, in principle, only be identified accurately once the velocities are known. This case is therefore more useful as a sensitive check on already existing calculations. For silver we have compared in this way the APW velocities with velocities derived from a Fourier model for the Fermi surface, the expansion coefficients being given by Halse. ${ }^{1}$ The result of this comparison, which seems to favor the Fourier model, is presented in Sec. X. In the concluding Sec. XI we comment on the best choice of the various experimental parameters and suggest some modifications of the experimental technique that will facilitate the data analysis and improve the accuracy.

\section{EXPERIMENTAL ARRANGEMENT}

The experiments were performed in a two-cavity transmission spectrometer with identical entrance and exit cavities, the sample serving as a common end wall. The cavities, with $Q=3000$, resonated in the $\mathrm{TE}_{112}$ mode, and the degeneracy was removed by Teflon pins mounted across the coupling holes. The resonant frequency of both cavities could be adjusted by inserting Teflon pins through holes at current nodes. This made it possible to correct for small unavoidable changes in resonant frequency during cooling to $4.2 \mathrm{~K}$. The cavities were surrounded by a can in order to exclude the liquid helium, and an exchange-gas pressure of typically 0.1 Torr was maintained in order to as sure thermal equilibrium. Any change in pressure was made at a very slow rate so as to avoid straining of the sample due to different pressure on its two sides. The magnetic field was generated by a superconducting solenoid provided with a separate superconducting modulation coil. The tilt angle between the magnetic field and the sample surface could be controlled and reproduced to within $1^{\prime}$ by tilting the solenoid. It was adjusted to zero by the aid of a very tilt-sensitive cyclotron-resonance signal.

The signal transmitted through the exit waveguide was detected in a superheterodyne receiver with 70-MHz intermediate frequency. A separate i.f. channel with a frequency discriminator served to lock the local-oscillator klystron to the signal klystron, and by detecting the phase difference between the two i.f. signals a voltage was obtained which was used for locking the signal klystron to the entrance cavity. The magnetic field was modulated at $270 \mathrm{~Hz}$, and the $270-\mathrm{Hz}$ component of the detected i.f. output was recovered in a PAR lock- 
in amplifier and fed to an $x-y$ recorder. The dis played signal was thus proportional to the magnetic field derivative of the signal emerging from the exit cavity.

\section{SAMPLE PREPARATION AND MOUNTING}

The starting material was in the form of disks, spark cut from a $\frac{3}{4}$-in. -diam Ag single crystal obtained from Metals Research, Inc. The initial resistivity ratio of around 350 was raised to 7500 by oxygen annealing, and the disks were then spark planed down to a thickness of $0.6 \mathrm{~mm}$. After a 60sec etching in dilute $\mathrm{HNO}_{3}$ in order to remove the deposits from the spark planing, the disks were chemically lapped on one side in concentrated $\mathrm{HNO}_{3}$ at a rate of $10-15 \mu / \mathrm{min}$. After a few minutes the sample surface would be specularly reflecting, although it would still have a fairly lustreless appearance. Microscopic inspection showed a large number of surface irregularities, and these could be removed by chemical polishing at a rate of 30 $\mu / \mathrm{min}$ in a solution consisting of $200 \mathrm{-g}$ saturated $\mathrm{CrO}_{3}$ solution, 25-ml $\mathrm{HNO}_{3}, 15-\mathrm{g} \mathrm{Na} \mathrm{SO}_{4}$, and 950- $\mathrm{ml} \mathrm{H}_{2} \mathrm{O}$. Both the lapping and the final polishing were performed on a Teflon cloth stretched over a stainless-steel plate. The surface would then appear flat, highly shiny, and with only a very small amount of orange-peel structure, the residual small-scale rounding being of the order of a few minutes of arc. After finishing one side, the sample was turned around, and the same procedure was applied to the second surface. During polishing the sample was mounted on a stainless-steel disk by means of paraffin. When the final thickness of $0.235 \mathrm{~mm}$ was reached, the sample was at the same time detached and cleaned by immersing in xylene and heating to $70^{\circ} \mathrm{C}$.

The polished sample was now mounted on the entrance cavity by means of a thin layer of paraffin. The second surface was covered by a thin piece of mylar foil, and the two cavities were lightly clamped together. With this arrangement the isolation between the cavities was limited to $60 \mathrm{~dB}$, so that a substantial leak signal would always be present. As shown in Sec. IV this leak signal serves as a reference with respect to which we measure the phase of the signal transmitted through the slab.

\section{DETECTION SCHEME}

The net signal at the input side of the i.f. mixer is the vector sum of the signal transmitted through the slab, the leak signal, and the local-oscillator signal. These may be written

$$
\begin{array}{ll}
E_{t}=E_{0} T e^{-i \omega t}, & T \equiv t e^{i \phi(B)}, \\
E_{l}=E_{0} L e^{-i \omega t}, & L \equiv l e^{i \psi}, \\
E_{10}=S e^{-i \omega_{10} t}, & S \equiv s e^{i \chi},
\end{array}
$$

where $\phi(B)$ is the magnetic-field-dependent phase of the signal transmitted through the slab; $\psi$ is the unknown, constant phase of the leak signal; and $\chi$ is the phase of the local-oscillator signal. $E_{0}$ is the incident amplitude, so $T$ is the transmission coefficient. The absolute value of the total field is then given by

$$
\left|E_{\text {tot }}\right|^{2}=\left|E_{0}\left(t e^{i[\phi(B)-\psi]}+l\right) e^{i \psi}+s e^{i\left(\omega_{1} . .^{t+\chi}\right)}\right|^{2},
$$

where

$$
\omega_{1 . t .} \equiv \omega-\omega_{10} \text {. }
$$

Introducing

$$
\xi e^{i \gamma} \equiv t e^{i(\phi(B)-\psi)}+l,
$$

we get

$$
\left|E_{\text {tot }}\right|^{2}=s^{2}+E_{0}^{2} \xi^{2}+2 s E_{0} \xi \cos \left(\omega_{1 . t .} t+\chi-\psi-\gamma\right) .
$$

Making use of the fact that $E_{0} t \ll E_{0} l \ll s$, and assuming the response of the diode to be given by $V_{\text {i.f. }} \sim\left|E_{\text {tot }}\right|^{\alpha}$, the amplitude of the i.f. voltage becomes

$$
\begin{aligned}
V_{\text {i.t. }} & \sim \alpha s^{\alpha-1} E_{0} \xi \\
& \sim E_{0}\left\{t^{2}+l^{2}+2 t l \cos [\phi(B)-\psi]\right\}^{1 / 2} \\
& \sim E_{0}\left[l+\operatorname{Re}\left(T e^{-i \psi}\right)\right] .
\end{aligned}
$$

Finally, by modulating the magnetic field, we measure

$$
\frac{d V_{1 . \ell}}{d B} \sim E_{0} \frac{d}{d B} \operatorname{Re}\left(T e^{-i \psi}\right) .
$$

In what follows we will occasionally drop constant phases from $T$, considering them as included in the unknown phase $\psi$.

\section{EXPERIMENTAL RESULTS}

The results of experiments with the sample nor mal along [0I1] and the magnetic field $\overrightarrow{\mathrm{B}}$ along [100] and [011], respectively, are shown in Figs. 1 and 2. For $\vec{B}$ along [100] we see a very complicated first-order size-effect line. The position of

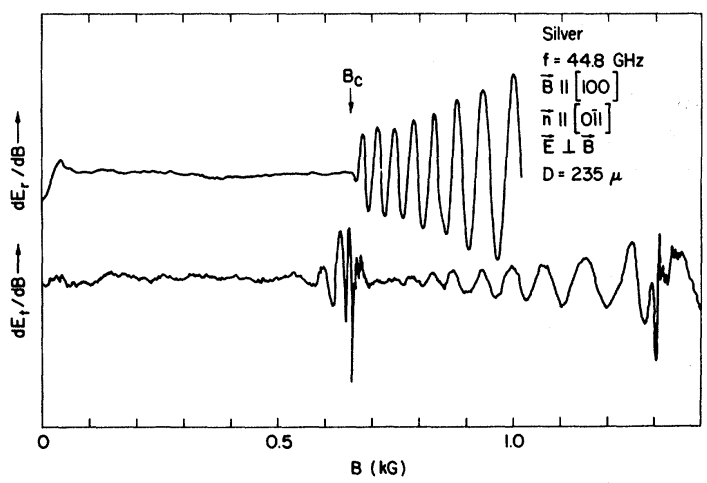

FIG. 1. Experimental reflection and transmission spectra for $\vec{B} \|[100]$. 


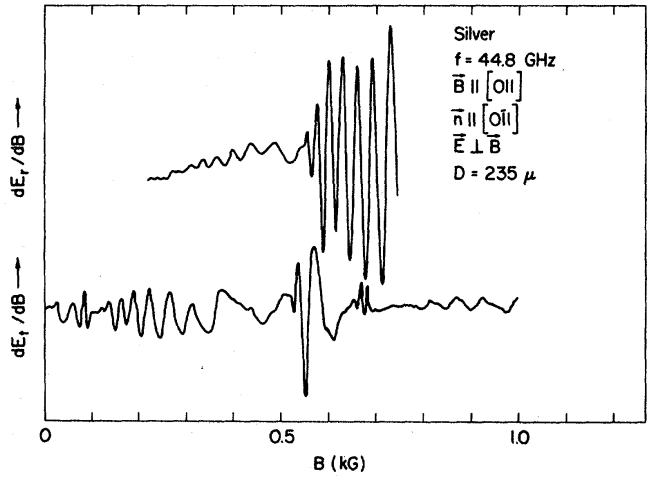

FIG. 2. Experimental reflection and transmission spectra for $\vec{B} \|[011]$. The oscillatory signal below cutoff in the reflection spectrum is cyclotron resonance from an incomplete dog's-bone orbit (orbit e in Fig. 3).

the cutoff field $B_{c}$, where the maximum orbit diameter equals the sample thickness, can be found to within $\pm 2 \%$ by comparing with the cyclotronresonance cutoff in the reflection spectrum. This follows since electrons at the central Fermi-surface section (orbit a in Fig. 3) are simultaneously extremal with respect to orbit diameter and cyclotron mass. Having thus located $B_{c}$ it appears that there is a substantial amount of structure on both sides. This is different from what is observed in size-effect experiments at frequencies $\omega$ small compared to the cyclotron frequency $\omega_{c}$, where the cutoff field corresponds to the onset of the sizeeffect line with no structure appearing below. ${ }^{5}$

Above $B_{c}$ we find a large number of narrow lines which become even more pronounced when the modulation amplitude is reduced. The origin of this structure is believed to be a beating of the extremal orbit diameter with successive oscillations of the skin layer at the two sample surfaces. This is supported by the fact that the relative width of the individual lines agree in order of magnitude with $\delta / D$, where $\delta$ is the skin depth and $D$ the sample thickness. The existance of such narrow lines suggests that the orbit diameter is very uniform over a wide section of the Fermi surface. This feature, which is confirmed by calculations, ${ }^{4}$ also accounts for the presence of a large number of higher-order size-effect lines created by reexcitation of the skin layer at integral multiples of the orbit diameter below the surface. A detailed analysis is complicated by the presence on the Fermi surface of two noncentral orbit minima in addition to the central maximum. Further, the nonuniform sample thickness caused by the over-all rounding of the surfaces will lead to signals corresponding to the minimum as well as the maximum sample thickness.

In the following we shall concentrate our effort on the range below $B_{c}$, where we observe a series of oscillations that can be traced down to $B / B_{c}$ $\simeq 0$. 7. Considering their amplitude it seems unlikely that they are caused by the small number of rosette orbits or by electrons from the limitingpoint zone of the Fermi surface. We shall see, in fact, that they are due to belly electrons which collide with both sample surfaces, but which are nevertheless able to carry a significant amount of power across the slab and deliver it to the exit cavity. The recorder tracing also contains the cyclotron-resonance signal from the belly electrons. This is associated with the leak signal, and $l$ of (4.5) thus has some magnetic field dependence in it. However, for the region of interest, below $B_{c}$, there are no cyclotron-resonance oscillations in this direction, so $d l / d B$ may be taken as zero, as in (4.6). In any event, since cyclotron resonance is a small perturbation of the surface impedance, we can always regard $l$ as being constant. In Sec. VI we show that the combined effect of the belly electrons and the leak signal can account for the qualitative features of the observed oscillations. Before we do this, however, let us review the [011] results.

For $\vec{B}$ along [011] we find a first-order size-effect line, but this time with much less structure. Now the size-effect electrons (orbit b in Fig. 3) have a minimum orbit diameter. They are located in two narrow, noncentral, Fermi-surface sections close to the necks, and, owing to the fairly rapid variation of orbit diameter with momentum component along $\vec{B}$, the higher-order size-effect lines are absent. The position of the size-effect field in this case cannot be inferred from the cyclotronresonance cutoff. The electrons with extremal cyclotron mass (orbit $c$ in Fig. 3) are located closer to the center of the Fermi surface, so that they

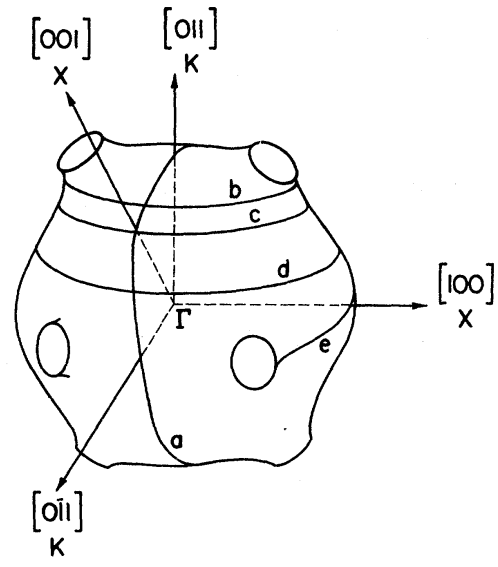

FIG. 3. Fermi surface of silver indicating various orbits referred to in the text. 


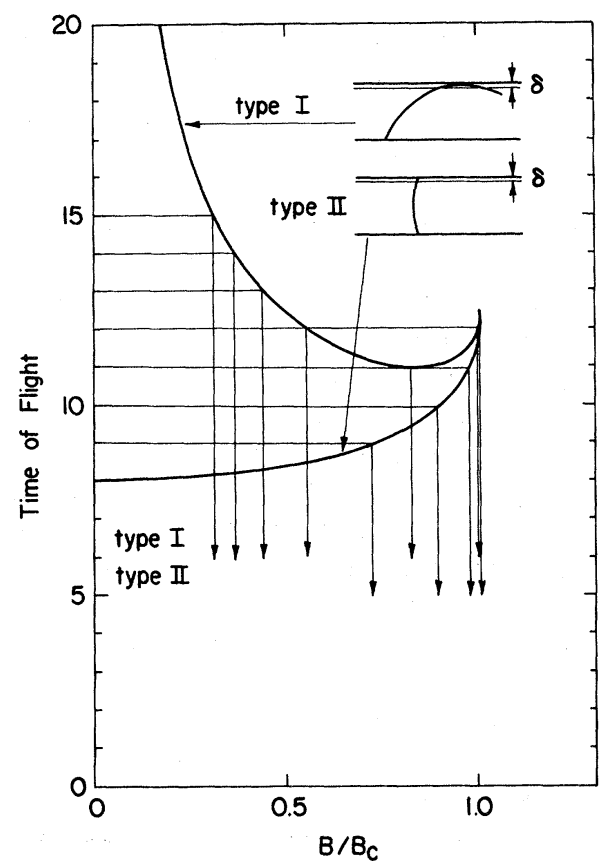

FIG. 4. Time of flight (in units of the microwave period) across the sample-vs-normalized magnetic field for circular type-I and type-II orbits. The arrows indicate magnetic fields at which the time of flight is an integral number of microwave periods.

have a larger orbit diameter, and the cyclotronresonance cutoff will therefore be at a higher field. However, present band calculations are so reliable that we can safely calculate $B_{c}$. Again we see a substantial amount of structure below $B_{c}$, but this time it extends towards lower magnetic fields, and the spacing of the peaks decreases rather than increases when $B$ is lowered. We shall show that we are here dealing with a transmission effect caused by electrons that collide with one surface, and we proceed now to present a simple model that can account for the results obtained in both directions.

\section{QUALITATIVE EXPLANATION}

Let us consider an electron moving in a circular trajectory, skimming the skin layer at one surface and colliding with the second as shown in Fig. 4. We shall denote this a type-I trajectory. The phase delay experienced by the electron during its time of flight $t_{I}$ across the sample is given by

$$
\Phi_{I}=\omega t_{I}=(2 C / \eta) \arcsin \sqrt{\eta},
$$

where $C=B_{\mathrm{cr}} / B_{c}$ is the ratio between the magnetic field at cyclotron resonance and at the size effect and $\eta=B / B_{c}$ is a variable, proportional to $B$ and normalized to unity at the size effect. If the field transmitted by the electron beats with a reference signal of fixed phase, we may expect a series of oscillations with a period determined by $\Delta \Phi_{I}=2 \pi$. Figure 4 shows that the qualitative behavior of such a signal closely resembles that observed for $\vec{B}$ along [011].

The [100] signal may be understood by reference to a slightly different situation. Let the electron collide with both sample surfaces, moving in what we shall call a type-II trajectory (Fig. 4). If a net signal is going to be produced, it must come from those orbits that have an extremal phase delay. These orbits are symmetric about the symmetry plane of the sample, and the phase delay is then given by the expression

$$
\Phi_{I I}=\omega t_{I I}=(2 C / \eta) \arcsin \eta .
$$

This time we find that the number of oscillations is limited and that they occur only close to $\eta=1$ (Fig. 4). This is exactly what we observe with $\vec{B}$ along [100]. It remains to be explained why two different kinds of trajectories have to be invoked in order to explain the results in the two directions. We shall deal with this problem in the more rigorous treatment that will be presented in Sec. VII.

\section{CALCULATION OF THE TRANSMISSION COEFFICIENT}

\section{A. Transmission Coefficient}

We consider our sample to lie parallel to the $x y$ plane, with one face at $z=0$ and the second face at $z=D$. The incident, reflected, and transmitted beams are expressed in terms of the electric field, which we take linearly polarized in the $y$ direction:

$$
\begin{aligned}
E(z, t) & =E_{0} e^{-i \omega t}\left(e^{i k z}+R e^{-i k z}\right), & & z \leqslant 0 \\
& =E_{0} e^{-i \omega t} T e^{i k(z-D)}, & & z \geqslant D .
\end{aligned}
$$

Here $k=\omega / c$ is the free space wave number, and $c$ is the speed of light.

We can express the transmission coefficient $T$ in terms of the parameters $\alpha$ and $\beta$ which give the boundary conditions at the two surfaces:

$$
\frac{d E}{d z}(0)=\alpha E(D)-\beta E(0), \quad \frac{d E}{d z}(D)=\beta E(D)-\alpha E(0) .
$$

The symmetry of the relations (7.2) is a consequence of the assumed inversion symmetry of the Fermi surface. Inserting (7.1) in (7.2) we immediately obtain the desired expression

$$
T=\frac{-2 i k \alpha}{(\beta-i k)^{2}-\alpha^{2}} .
$$

We shall see below that $\beta$ is essentially the inverse of the skin depth and is, consequently, much larger than $k$. We may therefore neglect $k$ in the denominator of (7.3). Further, an estimate based on circular orbits shows that $\alpha^{2} \leqslant \frac{1}{25} \beta^{2}$. The appearance of $\alpha^{2}$ in the denominator corresponds to 
the possibility of the field being propagated across the sample $3,5,7, \ldots$ times. These multiple reflections should then show up as additional peaks in the observed spectrum, with a magnitude of no more than $10 \%$ of the fundamental oscillations. Since such oscillations are not observed, we may be confident that the above estimate of the magnitude of $\alpha^{2}$ is not exceeded, and thus also neglect the $\alpha^{2}$ in the denominator of (7.3). We then have

$$
T \simeq-2 i(\omega / c) \alpha / \beta^{2} .
$$

\section{B. Variational Expression for $T$}

We may now derive variational expressions for $\alpha$ and $\beta$, and thus for $T$. The quantity

$$
A[E] \equiv \int_{0}^{D} d z\left[\left(\frac{d E}{d z}\right)^{2}-\frac{4 \pi i \omega}{c^{2}} E(z) j(z)\right]
$$

is stationary with respect to variations of $E(z) .{ }^{6-8}$ Here, the current is given by

$$
j(z)=\int_{0}^{D} d z^{\prime} K\left(z, z^{\prime}\right) E\left(z^{\prime}\right),
$$

where, since the Fermi surface has inversion symmetry, the kernel obeys

$$
K\left(D-z, D-z^{\prime}\right)=K\left(z, z^{\prime}\right) \text {. }
$$

The stationary value of $A[E]$ is achieved when $E(z)$ satisfies Maxwell's equations, and then (7.2) implies that

$$
A_{\text {stat }}=\left[E^{2}(D)+E^{2}(0)\right] \beta-2 E(0) E(D) \alpha,
$$

where $A_{\text {st at }}$ is the stationary value of $A[E]$.

If we choose the normalization $E(0)=1$, we can always write the electric field as where

$$
E(z)=f(z)+\rho f(D-z),
$$

$$
f(0)=1, f(D)=0 .
$$

Using this form for $E(z)$ in (7.5) combined with (7.8), we obtain variational expressions for $\alpha$ and $\beta$ :

$$
\begin{aligned}
& \beta=\int_{0}^{D} d z\left[\left(\frac{d f(z)}{d z}\right)^{2}\right. \\
& \left.\quad-\frac{4 \pi i \omega}{c^{2}} f(z) \int_{0}^{D} d z^{\prime} K\left(z, z^{\prime}\right) f\left(z^{\prime}\right)\right], \\
& \alpha=\int_{0}^{D} d z\left(-\frac{d f(D-z)}{d z} \frac{d f(z)}{d z}\right. \\
& \left.+\frac{4 \pi i \omega}{c^{2}} f(D-z) \int_{0}^{D} d z^{\prime} K\left(z, z^{\prime}\right) f\left(z^{\prime}\right)\right) .
\end{aligned}
$$

Neglecting terms of order $e^{-D / 6}$ ( $\delta$ is the skin depth), the upper limits in (7.11) may be extended to infinity to give

$$
\beta \simeq-\left(4 \pi i \omega / c^{2}\right) 1 / Z_{\infty} \equiv 1 / \delta_{\infty},
$$

where $Z_{\infty}$ and $\delta_{\infty}$ are, respectively, the surface impedance and skin depth of a semi-infinite sample. Thus, (7.4) can be rewritten

$$
T \simeq-2 i(\omega / c) \delta_{\infty}^{2} \alpha .
$$

To the same accuracy, we may neglect the first term in (7.12) and get

$$
\alpha=\left(4 \pi i \omega / c^{2}\right) \int_{0}^{D} d z f(D-z) \int_{0}^{D} d z^{\prime} K\left(z, z^{\prime}\right) f\left(z^{\prime}\right) .
$$

\section{Evaluation of $T$}

We can obtain an expression for the kernel $K\left(z, z^{\prime}\right)$ in (7.6) from the Chambers expression for the current ${ }^{9}$ (assuming diffuse reflection at the sample surfaces) and use this, together with (7. 14) and (7.15), to write the transmission coefficient as

$$
T=\frac{2}{\pi^{2}} \frac{\omega^{2} e^{2}}{c^{2}} \delta_{\infty}^{2} \int d k_{x} \frac{m_{c}}{\omega_{c}} I\left(k_{x}\right),
$$

where

$$
\begin{aligned}
I\left(k_{x}\right)= & \int_{0}^{D} d z f[D-z] \int_{0}^{2 \pi} d \phi v_{y}\left(\phi, k_{x}\right) \\
& \times \int_{\lambda\left(z ; \phi, k_{x}\right)}^{\phi} d \phi^{\prime} e^{i \nu\left(\phi-\phi^{\prime}\right)} v_{y}\left(\phi^{\prime}, k_{x}\right) \\
& \times f\left[z-R(\phi)+R\left(\phi^{\prime}\right)\right]
\end{aligned}
$$

Here $-e$ is the electronic charge; $k_{x}$ is the component of momentum parallel to the magnetic field; $m_{c}=e B / \omega_{c} c$ is the cyclotron mass and a function of $k_{x} ; \phi$ and $\phi^{\prime}$ are $\omega_{c}$ times time variables which give the position of the electron on its orbit (at fixed $k_{x}$ ) about the Fermi surface, with $\phi=0$ at the "top" of the orbit where the $z$ component of the velocity, $v_{z}\left(\phi, k_{x}\right)$, vanishes. The $y$ component of the electron's velocity is $v_{y}\left(\phi, k_{x}\right)$,

$$
\nu=(\omega+i / \tau) / \omega_{c},
$$

where $\tau$ is the relaxation time, and

$$
R(\phi)=\left(1 / \omega_{c}\right) \int_{0}^{\phi} v_{z}\left(\phi^{\prime}, k_{x}\right) d \phi^{\prime} .
$$

Finally, $\lambda\left(z ; \phi, k_{x}\right)$ is the last "time" previous to $\phi$ at which an electron collided with a surface if at $\phi$ the electron is at a depth $z$.

It is the presence of $\lambda$ which necessitates the separate consideration of the contributions from type-I and type-II trajectories (Fig. 4). In the course of the calculations we shall make use of a set of assumptions, all of which are well satisfied by the experimental conditions. They are

$$
|\delta| / R_{c} \ll 1, \omega_{c} \tau ;|\delta| / D \ll 1 ;|\nu \delta| / R_{c} \ll 1 .
$$

Here $R_{c}$ is a typical cyclotron radius. For the type-II oscillations we further require $|\nu| \gg 1$. Also, our results will be restricted to magnetic fields not too close to $B_{c}$ [for our case we require $\left.\left(B_{c}-B\right) / B_{c} \gg 10^{-3}\right]$. We shall not attempt to calculate $E(z)$, but rather take advantage of the stationary nature of (7.16) and use the trial function 


$$
f(z)=e^{-z / 0},
$$

with $\delta$ ultimately taken equal to $\delta_{\infty}$.

\section{Contribution of Type-I Trajectories}

There are two kinds of type-I trajectories, those that skim the $z=0$ surface and those that skim the $z=D$ surface. Due to the inversion symmetry of the Fermi surface these give the same contribution when integrated over $k_{x}$. Hence, we will only consider the contribution from those skimming the $z=0$ surface, and include a factor of 2 to account for the others.

The type-I contribution to (7.17), called $I_{1}\left(k_{x}\right)$, has the same form as (7.17) except that the upper limit in the $\phi$ integration is now $\phi_{\varepsilon}$, defined by

$$
z=R\left(\phi_{z}\right) \text {. }
$$

Using (7.21) and the fact that $\delta$ is much smaller than the cyclotron radius, the $\phi^{\prime}$ integration is performed by stationary phase in the usual way. However, because we deal with $|\nu| \sim 25$, we cannot neglect retardation effects ${ }^{10}$ occurring because the microwave phase changes while the electron passes through the skin depth. This means that the stationary point is not where $v_{z}=0$, but rather at $\phi_{c}$ which satisfies the "surf-riding" condition:

$$
v_{z}\left(\phi_{c}\right)=-i \nu \omega_{c} \delta \text {. }
$$

The $\phi^{\prime}$ contour must be distorted to pass through the complex $\phi_{c}$, and then the limits may be extended to infinity to give

$$
\begin{aligned}
I_{1}\left(k_{x}\right)= & 2\left(2 \pi \delta \omega_{c}\right)^{1 / 2}\left(\frac{\partial v_{z}}{\partial \phi}\left(\phi_{c}, k_{x}\right)\right)^{-1 / 2} \\
& \times v_{y}\left(\phi_{c}\right) F\left(k_{x}\right) e^{-i \nu \phi_{c}-(1 / 8) R\left(\phi_{c}\right)} e^{-D / 8},
\end{aligned}
$$

where

$F\left(k_{x}\right)=\int_{0}^{D} d z \int_{0}^{\Phi} d \phi v_{y}\left(\phi, k_{x}\right) e^{i \nu \phi} e^{(1 / \delta) R(\phi)}$.

Using (7.22) to change variables from $z$ to $\phi_{z}$, and integrating by parts, we get

$$
F\left(k_{x}\right)=\int_{0}^{\phi} D d \phi[D-R(\phi)] v_{y}\left(\phi, k_{x}\right) e^{i \nu \phi} e^{(1 / \delta) R(\phi)} .
$$

This last integral is integrated after expanding the integrand around $\phi=\phi_{D}$. Then, neglecting higher powers of $\delta$ divided by the cyclotron radius, we get the result

$$
\begin{array}{r}
I_{1}\left(k_{x}\right)=2(2 \pi)^{1 / 2} \delta^{5 / 2} \omega_{c}^{3 / 2} \frac{v_{y}\left(\phi_{D}, k_{x}\right)}{v_{z}\left(\phi_{D}, k_{x}\right)} \\
\times \frac{e^{i \nu \Phi_{D}}}{1+i \nu \delta \omega_{c} / v_{z}\left(\phi_{D}, k_{x}\right)} G\left(k_{x}\right),
\end{array}
$$

where

$$
G\left(k_{x}\right)=\frac{v_{y}\left(\phi_{c}, k_{x}\right)}{\left[\partial v_{z}\left(\phi_{c}, k_{x}\right) / \partial \phi\right]^{1 / 2}} \frac{e^{-i \nu \phi_{c}-(1 / \delta) R\left(\phi_{c}\right)}}{1+i \nu \delta \omega_{c} / v_{z}\left(\phi_{D}, k_{x}\right)} .
$$

To simplify the bookkeeping, we have assumed only one stationary point in the integration. That is, we assumed a convex orbit.

The most important part of (7.27) is the $e^{i \nu \Phi_{D}}$ Here $\phi_{D}$ is the "time" it takes an electron, which skims the $z=0$ surface, to reach the $z=D$ surface, and it is determined, according to (7.22), by $D$ $=R\left(\phi_{D}\right)$. Then the real part of $\nu \phi_{D}$ is the phase delay $\phi_{I}$ of (6.1) in more general form. The imaginary part describes the damping caused by the bulk collisions.

The amplitude factors in (7.27), however, are not negligible. The two factors of $v_{y}$ arise from the coupling with the electric field (in the $y$ direction) at both surfaces. $v_{y}\left(\phi_{D}\right)$ passes through zero, producing a zero in $I_{1}$ and a phase change of $\pi$, at that value of $\phi_{D}$ for which the type-I orbit hits the $z=D$ surface normally (at $\phi_{D}=\frac{1}{2} \pi$ for circular or bits and for orbits of fourfold symmetry). This reflects the fact that an electron coming perpendicular to the surface cannot contribute to the microwave field parallel to the surface.

The appearance of $\phi_{c}$ in $G\left(k_{x}\right)$ reflects retardation effects (in the absence of which $\phi_{c}$ vanishes). The factors of $\delta$ were discussed in Ref. 3. They reflect the number of type-I orbits contributing and the amount of time they spend in the skin layer.

\section{Contribution of Type-II Trajectories}

To evaluate the contribution of type-II trajectories, we return to (7.17). The type-II contribution is called $I_{2}\left(k_{x}\right)$ and is of the same form as (7. 17) except that the $\phi$ integration runs from $\phi_{z}$ to $\phi_{m}\left[\phi=\phi_{m}\right.$ at the "bottom" of the orbit, where $v_{z}\left(\phi, k_{x}\right)$ vanishes]. The $\phi^{\prime}$ integration is per formed after expanding the integrand around $\phi^{\prime}$ $=\lambda$. Taking advantage of the fact that $|\nu| \gg 1$, the $\phi$ integration is performed by stationary phase, the stationary point being at $\phi=\phi_{s}^{z}$ and

where

$$
\lambda\left(z ; \phi_{s}^{z}, k_{x}\right)=\lambda_{s}^{z}
$$

$$
v_{z}\left(\lambda_{s}^{z}\right)=v_{z}\left(\phi_{s}^{z}\right)
$$

and

$$
z=R\left(\phi_{s}^{z}\right)-R\left(\lambda_{s}^{z}\right) .
$$

Finally, (7.22) is used to change variables from $z$ to $\phi_{z}$, and expanded the integrand near $\phi_{z}=\phi_{D}$ (that is, near $z=D$ ). The result is

$$
\begin{aligned}
I_{2}\left(k_{x}\right)=e^{i \pi / 4}\left(\frac{2 \pi}{\nu}\right)^{1 / 2} \delta^{2} \omega_{0} \frac{v_{y}\left(\phi_{s}^{D}, k_{x}\right) v_{y}\left(\lambda_{s}^{D}, k_{x}\right)}{v_{z}\left(\phi_{s}^{D}, k_{x}\right)\left(-d^{2} \lambda / d \phi^{2}\right)_{s}} \\
\\
\quad \times \frac{e^{i \nu\left[\phi_{s}^{D}-\lambda_{s}^{D}\right.}}{\left(1+i \nu \delta \omega_{c} / v_{z}\left(\phi_{s}^{D}, k_{x}\right)\right)^{2}} \cdot \quad(7.30)
\end{aligned}
$$

Here $\phi_{s}^{D}$ and $\lambda_{s}^{D}$ are defined by (7.28) and (7.29) with $z=D$, and 


$$
-\left.\frac{d^{2} \lambda}{d \phi^{2}}\right|_{s}=\frac{1}{v_{z}\left(\phi_{s}^{D}, k_{x}\right)}\left(\frac{\partial v_{z}}{\partial \phi}\left(\lambda_{s}^{D}, k_{x}\right)-\frac{\partial v_{s}}{\partial \phi}\left(\phi_{s}^{D}, k_{x}\right)\right)
$$

The most important part of (7.30) is the $\exp [i \nu$ $\left.\times\left(\phi_{s}^{D}-\lambda_{s}^{D}\right)\right]$, with the real part of $\nu\left[\phi_{s}^{D}-\lambda_{s}^{D}\right]$ being the generalization of the phase delay $\phi_{\text {II }}$ of (6.2). The amplitude factors, similar in form to those in $I_{1}\left(k_{x}\right)$, are again non-negligible. There are no retardation effects here, due to the shorter time that type-II electrons spend in the skin layer. For the same reason the contribution of each individual electron is smaller than in the type-I case, but this is made up for by the fact that there are more type-II than type-I trajectories. This accounts for the different factors of $\delta$ and $\nu$ as discussed in Ref. 3.

\section{D. $k_{x}$ Integration}

One must still perform the integration over $k_{x}$ in (7. 16). The way in which this is done depends on the shape of the Fermi surface, and for $\mathrm{Ag}$ we shall need to use two different techniques for the two different directions of the magnetic field.

When $\vec{B} \|[100]$ the Fermi surface has very little variation with $k_{x}$, and the $k_{x}$ integration may be simply replaced by a factor corresponding to the length, in the $k_{x}$ direction, of the relevant part of the Fermi surface. Here, as discussed in Ref. 3, the type-II orbits give the largest contribution, so that the transmission coefficient can be considered as just proportional to (7.30).

For $\overrightarrow{\mathrm{B}} \|[011]$, however, there is a definite $k_{x}$ dependence, so the integration selects that value of $k_{x}$ (if any) which gives $I_{1}\left(k_{x}\right)$ or $I_{2}\left(k_{x}\right)$ a stationary phase. The phase of $I_{1}\left(k_{x}\right)$ is stationary when (ignoring the retardation corrections, which produce a negligible effect over the region of interest)

$$
\begin{aligned}
0= & \frac{1}{\omega_{c}} \frac{d \omega_{c}}{d k_{x}} \phi_{D}-\frac{d \phi_{D}}{d k_{x}} \\
= & \frac{1}{\omega_{c}} \frac{d \omega_{c}}{d k_{x}}\left(\phi_{D}-\frac{\omega_{c} D}{v_{z}\left(\phi_{D}, k_{x}\right)}\right) \\
& \quad+\frac{1}{v_{z}\left(\phi_{D}, k_{x}\right)} \int_{0}^{\phi_{D}} d \phi \frac{\partial v_{z}}{\partial k_{x}}\left(\phi, k_{x}\right),
\end{aligned}
$$

and the stationary-phase integration provides a factor of

$$
e^{i \pi / 4}(2 \pi / \nu)^{1 / 2}\left[\omega_{c}\left|\frac{d^{2}}{d k_{x}^{2}}\left(\frac{1}{\omega_{c}} \phi_{D}\right)\right|\right]^{-1 / 2}
$$

evaluated at the stationary point (7.32). The phase of $I_{2}\left(k_{x}\right)$ is stationary when

$$
\begin{aligned}
0 & =\frac{1}{\omega_{c}} \frac{d \omega_{c}}{d k_{x}}\left(\phi_{s}^{D}-\lambda_{s}^{D}\right)-\frac{d}{d k_{x}}\left(\phi_{s}^{D}-\lambda_{s}^{D}\right) \\
& =\frac{1}{\omega_{c}} \frac{d \omega_{c}}{d k_{x}}\left(\phi_{s}^{D}-\lambda_{s}^{D}-\frac{\omega_{c} D}{v_{z}\left(\phi_{s}^{D}, k_{x}\right)}\right)
\end{aligned}
$$

$$
+\frac{1}{v_{z}\left(\phi_{s}^{D}, k_{x}\right)} \int_{\lambda_{s}}^{\phi} d \phi \frac{\partial v_{z}}{d k_{x}}\left(\phi, k_{x}\right)
$$

while the integration provides a factor of

$$
e^{i \pi / 4}(2 \pi / \nu)^{1 / 2}\left[\omega_{c}\left|\frac{d^{2}}{d k_{x}^{2}}\left(\frac{1}{\omega_{c}}\left(\phi_{s}^{D}-\lambda_{s}^{D}\right)\right)\right|\right]^{-1 / 2}
$$

evaluated at the stationary point (7.34). If both type trajectories contribute, their contributions must be added. We should point out that whenever the Fermi surface is symmetric about a given orbit, that orbit will automatically satisfy both (7.32) and (7.34). This is not the case, however, for $\vec{B} \|[011]$, because of the presence of the necks at the center of the Fermi surface.

\section{COMPARISON WITH EXPERIMENT}

$$
\text { A. } \vec{B} \|[100]
$$

We are now ready to compare our results with experiment. Using (4.6), (7.16), and the results of Sec. VII D, the experimental curve should be proportional to

$$
\frac{d}{d B} \operatorname{Re}\left(\frac{e^{-i \varphi} I_{2}\left(k_{x}=0\right)}{\omega_{c}}\right),
$$

where the constant phase $\zeta$ includes the unknown leak phase $\psi$, as well as constant phases associated with the electromagnetic field. We have treated $\zeta$ as an adjustable parameter and used (7. 30) for $I_{2}\left(k_{x}\right)$. We used $m_{c}$ and the Fermi velocities as calculated in an APW calculation of the properties of Ag. ${ }^{4}$ These calculated masses and velocities have proven to be within $1 \%$ of the experimental values. We neglected the rosette orbits as they give rise to cyclotron-resonance signals which are orders of magnitude weaker than those of the belly electrons. Further, they cause no observable effect in the relevant range of magnetic field.

In Fig. 5 we plot the zero intercepts of (8.1) as a function of $\zeta$. It is seen that we can choose a value of $\zeta$ that yields quite excellent agreement with the experimental observations. As an indication of the quality of this agreement, we have replotted this curve in Fig. 6 in an "extended-zone" representation. Here we have used the fact that the zeros of (8.1) are unchanged if $\zeta$ is displaced by $\pi$, and connected all the lines of Fig. 5 together. On the same curve we have also plotted (8.1) for the case of circular orbits. There is no choice of $\zeta$ that will allow agreement between the circular results and experiment. The rather marked difference between the results for the actual orbits and for circular orbits is caused by deviations from isotropy of less than $5 \%$, and this suggests that a suitable analysis of the experimental data may give rather precise information about the an- 


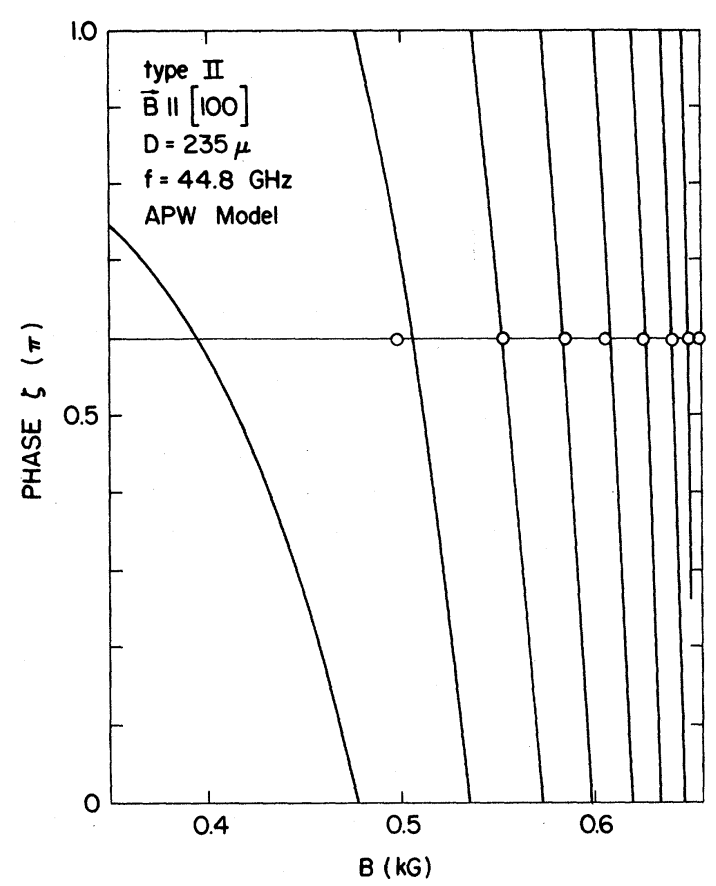

FIG. 5. Zero intercepts of transmitted signal as a function of the over-all phase $\zeta$ (in units of $\pi$ ). Best agreement with the experimental points (circles) is obtained for $\zeta=0.6 \pi$.

isotropy. We shall return to this in Sec. IX. The calculated signal, using (8.1) and the actual orbits, for the best value of $\zeta=0.60 \pi$, is shown in Fig. 7 .

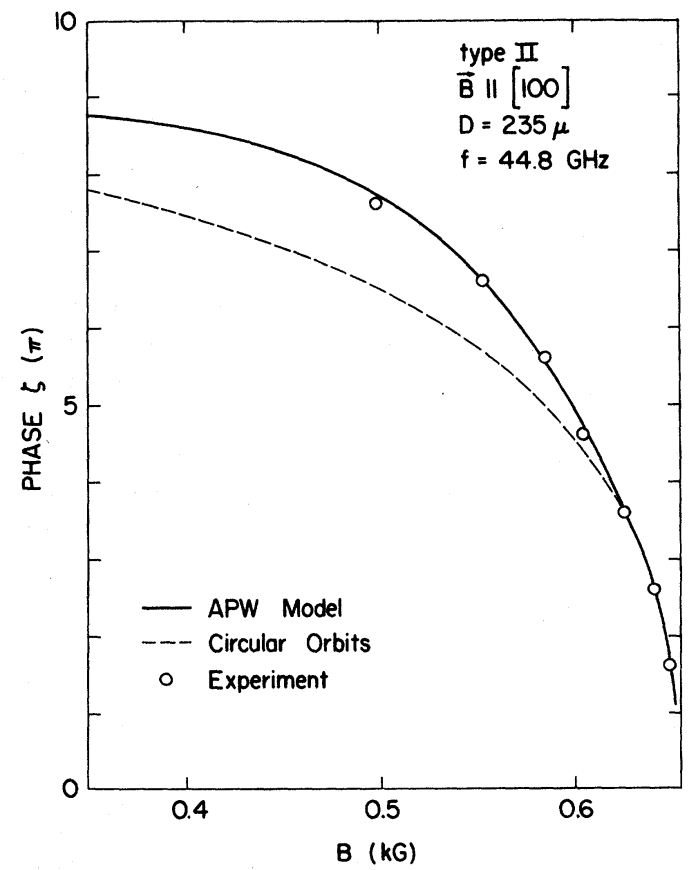

FIG. 6. Extended-zone plot of Fig. 5. This plot is essentially equivalent to the lower curve of Fig. 4.

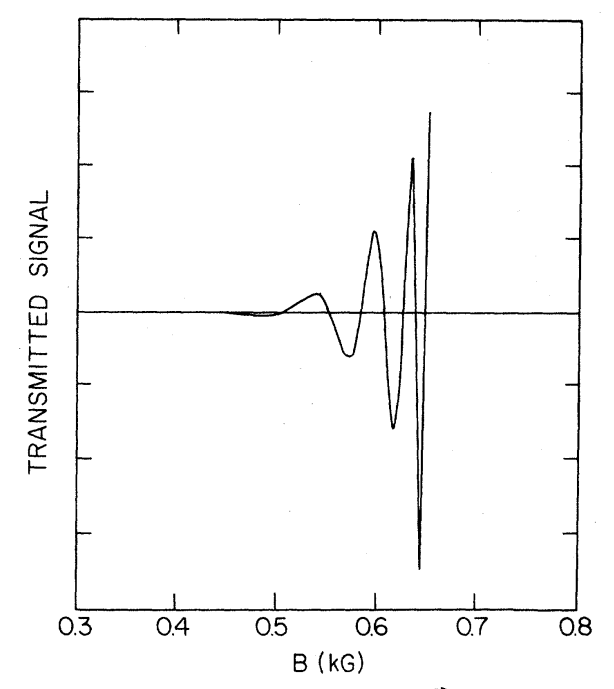

FIG. 7. Transmission spectrum for $\overrightarrow{\mathrm{B}} \|[100]$ calculated from the APW model.

\section{B. $\overrightarrow{\mathbf{B}} \|[011]$}

In this direction, as indicated in Sec. VIID, we must find those values of $k_{x}$ which give stationary phases. Again using the APW velocities we have calculated those $k_{x}$ which satisfy (7.32) or (7.34) as a function of $B$. The results are shown in Fig. 8. The sensitivity of these curves is indicated by plotting on the same figure the results obtained under the assumption of circular orbits with the same $k_{x}$ dependence of the averaged Fermi-surface parameters. Note that the APW model predicts a second type-I stationary point below $100 \mathrm{G}$.

To obtain the experimentally observed curve, we must use (4.6) and (7.16), inserting the above

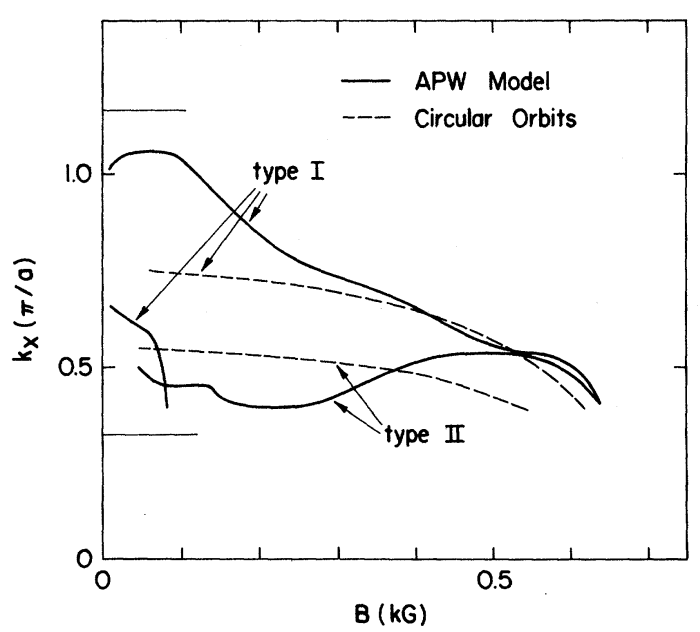

FIG. 8. Position of stationary point as a function of magnetic field for $\vec{B} \|[011]$. No precise Fermi-surface data are available for $k_{x}>1.16 \pi / a$ and $k_{x}<0.32 \pi / a(a$ $=4.069 \AA$ ). 


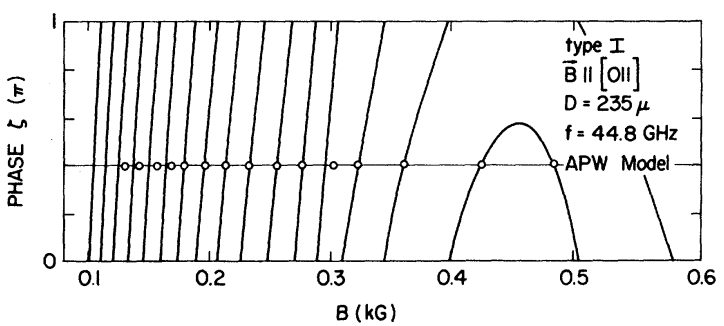

FIG. 9. Zero intercepts of transmitted signal as a function of the over-all phase $\zeta$ (in units of $\pi$ ) for the APW model. Best agreement with the experimental points (circles) is obtained for $\zeta=0.4 \pi$.

values of $k_{x}$ into the expressions for $I_{1}\left(k_{x}\right)$ and $I_{2}\left(k_{x}\right)$, and using the factors (7.33) or (7.35), as appropriate, for the $k_{x}$ integration. A glance at the experimental curve, Fig. 2, indicates the presence of oscillations with the type-I characteristic (see Fig. 4), so we will concentrate our attention on these oscillations first, and return to type-II later. In Fig. 9 we plot the zero intercepts of the calculated signal as a function of the over-all phase $\zeta$, and this time we find it possible to choose a value $\zeta=0.4 \pi$ which gives good agreement with the experiment for $180<B<500 \mathrm{G}$. [The point at 304 $G$ is very close to the value of $B$ at which the amplitude factor in (7.27) goes through zero, and its position is particularly sensitive to the Fermisurface parameters.] As above, we have replotted the curve in an extended-zone representation in Fig. 10, and compared it with the case of circular orbits. Although the difference is not as dramatic as in the type-II case (Fig. 6) (there the circularorbit velocities were determined from the size effect and the cyclotron mass, whereas here we have the freedom of fitting them to averages of the actual velocities), the data clearly show a preference for the noncircular results at both ends of the spectrum. Figure 11 shows the calculated signal corresponding to the best choice for $\zeta$. Given, then, this good agreement at intermediate mag-

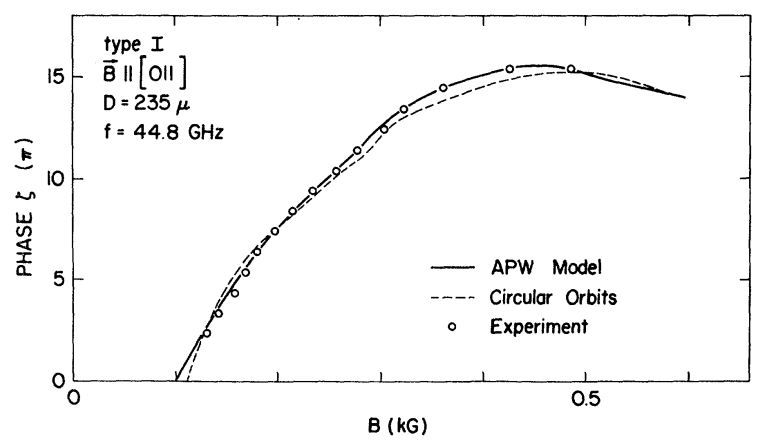

FIG. 10. Extended-zone plot of Fig. 9. This plot is essentially equivalent to the upper curve of Fig. 4.

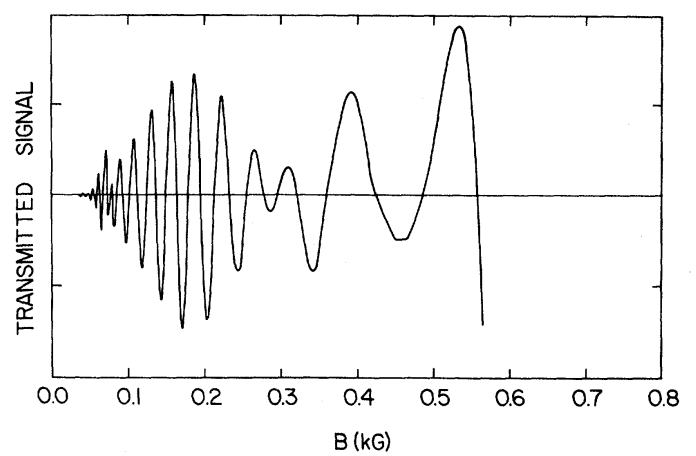

FIG. 11. Transmission spectrum for $\vec{B} \|[011]$ calculated from the APW model. The irregularity around $90 \mathrm{G}$ is caused by the appearance of the second stationary orbit on the Fermi surface (see Fig. 8).

netic fields, we must discuss the situation at the two extremes.

\section{Low Fields}

The difference in phase between the calculated and observed curves in the region $130<B<180 \mathrm{G}$ is believed to be related to our choice of Fermisurface model. As is seen from Fig. 8 , this is the region where the value of $k_{x}$ changes rapidly. The calculated curve then depends sensitively on the Fermi-surface parameters, and small errors can be expected to cause disagreement with the experimental curve. This suggestion is substantiated in Sec. $X$ where we carry through the calculations with a different Fermi-surface model.

The lack of agreement for $B<130 \mathrm{G}$ is probably due to two different effects which have not been included in our calculations. In the region $100<B$ $<130 \mathrm{G}$ the electron transit time through the skin layer is comparable to one microwave period, and retardation effects are then sensitive to details of the electric field. Calculations have shown that a change of trial field mainly affects the amplitude of the oscillations, but leaves the zero intercepts unchanged. The transmitted signal for a trial field $f(z) \sim \exp \left(-2 z / 3 \sqrt{3}\left|\delta_{\infty}\right|\right) \sin \left(2 z / 3\left|\delta_{\infty}\right|-\frac{1}{6} \pi\right)$, which is shown in Fig. 12, shows that one can indeed ob-

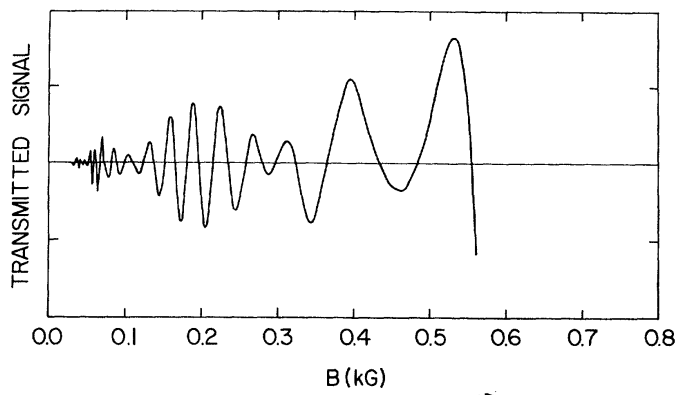

FIG. 12. Transmission spectrum for $\vec{B} \|[011]$ calculated from the APW model with a modified trial field. 


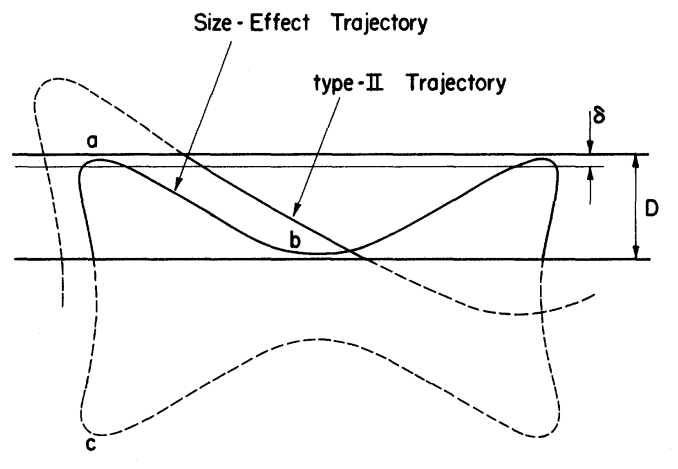

FIG. 13. Incomplete dog's-bone orbit, shown at two different magnetic fields, giving rise to size-effect and type-II oscillations. \{Cyclotron resonance from this orbit is present in Fig. 2 and has been discussed by Henningsen [J. O. Henningsen, Phys. Rev. B $\underline{4}, 3280$ (1971)].

tain the desired amplitude reduction in this way. ${ }^{11}$ The observed signal in the region $B<100 \mathrm{G}$ is probably caused by electrons moving on a part of a dog's-bone orbit (orbit e in Fig. 3). As seen in Fig. 13 these electrons may give rise to a size effect which should be located at $B=95 \mathrm{G}$. Since we are dealing with a central Fermi-surface section, we expect to find type-II oscillations at lower fields, and a quantitative estimate shows that the oscillations seen in the interval $0<B<100 \mathrm{G}$ can be explained in this way. We note that the relative spacing of the individual peaks is larger than found for the type-II signal of Fig. 7, and that they decay much more slowly with decreasing magnetic field. Both of these observations are explained by the shape of the orbits. In the limit $B \rightarrow 0$ the phase delay will depend only weakly on the magnetic field, and the impact angle will tend towards a constant value different from $\frac{1}{2} \pi$. We note that the size effect from the entire dog's-bone orbit (from points a to c in Fig. 13), which should occur near $B=360 \mathrm{G}$, is entirely unobservable, presumably because the orbit is much more sharply curved at point $c$ than at point $b$. Unfortunately we are unable to perform exact calculations for this orbit as we lack a reliable set of velocities for it.

\section{High Fields}

The situation here is complicated by the dependence on $k_{x}$ of the orbit diameter. Thus, although the electrons which are providing the type-I signal near $500 \mathrm{G}$ do not have their size effect until near $650 \mathrm{G}$, there are electrons with smaller orbit diameters. The electrons with the smallest orbit diameter (at $k_{x}=1.26 \pi / a$ ) will have their size effect at $B=505 \mathrm{G}$ if the thickness $D=235 \mu$. The onset of the observed line is at $B=517 \mathrm{G}$, corresponding to a diameter slightly larger than the minimum value. Due to the presence of these smaller orbits we have not concerned ourselves with fields greater than around $500 \mathrm{G}$. Our calculations should not be expected to be reliable in the vicinity of any size effect, where the electric fields in the skin layer become significantly dependent on the magnetic field.

We do, however, have two problems remaining to be explained. The first is the truncation of the peak near $400 \mathrm{G}$. This is rather difficult to account for. The small rapid signals near $430 \mathrm{G}$ may be an effect of the higher derivatives occurring in the factor (7.33). A slight trace of them appears in the calculated curve Fig. 11, but the calculated Fermi-surface parameters are not sufficiently accurate to try to examine this more closely. In any event, this would not account for the observed truncation of the peak. It is true that this region is quite close to the minimum in $\nu \phi_{D}$, which is reflected in the maximum in Fig. 10. (This can be verified experimentally using the variable-phase technique discussed in Sec. XI. A change in phase should cause the two zeros adjacent to this maximum to move in opposite directions.) It is therefore quite sensitive to Fermi-surface parameters, sample thickness, etc. However, it is hard to see how these effects can produce the observed truncation.

The truncation may be related to the other problem, which is the absence of observed type-II oscillations. While it is true that for $\vec{B} \|[100]$ the type-II oscillations do not extend below around 500 $\mathrm{G}$ (see Fig. 7), calculations for $\vec{B} \|[011]$ indicate that quite large, slow type-II oscillations should extend well below 400 G. ${ }^{12}$ No such oscillations are observed experimentally except, possibly, with greatly reduced amplitude, in the peak truncation near $400 \mathrm{G}$. We are at a loss to explain this. We do note that both the value of the stationary point $k_{x}$, as well as the distribution of type-II oscillations, depends sensitively on the details of the Fermi surface in a region somewhat near the necks $\left(k_{x} \simeq 0.5 \pi / a\right)$, where the surface has not been verified by other experiments. It should also be borne in mind that there is an experimental uncertainty associated with the orientation of the magnetic field relative to the crystal axis. However, although we have not explored the effects caused by small deviations from the symmetry directions, it does seem unlikely that this could cause such a dramatic discrepancy.

\section{EXTRACTION OF FERMI VELOCITIES}

As we have seen, the results from this kind of experiment are significantly changed by alterations in the Fermi velocities of only a few percent. This suggests that one might be able to use this approach to extract Fermi velocities to high precision. In this section we shall demonstrate how this can be 
done.

We saw in Sec. IV that what we measure is $\operatorname{Re}\left[\mathrm{Te}^{-i \psi}\right]$, where $\psi$ is some constant phase. (In Sec. IV we argued that we actually measure the magnetic field derivative of this quantity, but we shall see below that it can be measured directly.) What we are interested in is the $B$-dependent part of the phase of $T$, and it is therefore desirable to remove any significant $B$ dependence of the amplitude of $T$. This can be done by dividing out the amplitude factors, as expressed in (7.30) for example. These factors involve the velocities we are trying to determine, but for removal of the major $B$ dependence it is sufficient to use approximate values.

Having done this, we may assume that the remaining oscillations are due to the $B$ dependence of the phase of $T$. We will examine this phase for the two types of oscillations, combining all constant phases, such as $\psi$, various factors of $\pi$, etc., into one phase $\zeta_{i}(i=1,2)$. Then from (7.27) and (7.27a) the phase of the type-I contribution is

$$
F_{1}(B)=\left(\omega / \omega_{c}\right) \phi_{D}-\operatorname{Im}\left[i \nu \phi_{c}+(1 / \delta) R\left(\phi_{c}\right)\right]-\zeta_{1} .
$$

From (7. 30), the phase of the type-II contribution is

$$
F_{2}(B)=\left(\omega / \omega_{c}\right)\left(\phi_{s}^{D}-\lambda_{s}^{D}\right)-\zeta_{2} .
$$

We may ignore the $B$ dependence of the phase arising from the dependence of $k_{x}$ on $B$ because the phase is stationary with respect to $k_{x}$, and from (9.1) we then get

$$
\frac{1}{\omega B}\left(\frac{d}{d B}\left[B F_{1}(B)\right]+\zeta_{1}\right)=\frac{1}{\omega_{c}} \frac{\partial \phi_{D}}{\partial B} .
$$

Note that although retardation effects give a substantial contribution to $F_{1}(B)$ they do not enter in (9.3) since $\phi_{c}$ is independent of $B$. Differentiating $D=R\left(\phi_{D}\right)$ now gives

$$
0=-\frac{D}{B}+v_{z}\left(\phi_{D}, k_{x}\right) \frac{1}{\omega_{c}} \frac{\partial \phi_{D}}{\partial B}
$$

so that

$$
\frac{\omega D}{v_{z}\left(\phi_{D}, k_{x}\right)}=\frac{d}{d B}\left[B F_{1}(B)\right]+\zeta_{1} .
$$

Similarly, one obtains, from (9.2),

$$
\frac{\omega D}{v_{z}\left(\phi_{s}^{D}, k_{x}\right)}=\frac{d}{d B}\left[B F_{2}(B)\right]+\zeta_{2} \text {. }
$$

A knowledge of the phases $F_{i}(B)$ thus provides a measure of the velocities at the positions determined by $\phi_{D}$ or $\phi_{s}^{D}$, and $k_{x}$. We can locate these positions in $k$ space if we use the variable $k_{y}$ instead of $\phi$. We choose the origin in the $k_{y}-k_{z}$ plane according to $k_{z}=0$ at $v_{z}=0$ and $k_{y}=0$ at $v_{y}=0$ (assuming symmetric convex orbits), and let $k_{y}=K$ when $v_{z}=0\left(k_{z}=0\right)$. Then $(9.5)$ becomes $\frac{\omega D}{v_{z}\left[k_{y}=K-(e B D / \hbar c), k_{x}\right]}=\frac{d}{d B}\left[B F_{1}(B)\right]+\zeta_{1}$.

Likewise, we define

$K_{1}-K_{2}=e B D / \hbar c ; \quad v_{z}\left(k_{y}=K_{1}\right)=v_{z}\left(k_{y}=K_{2}\right)$,

and (9.6) becomes

$$
\frac{\omega D}{v_{z}\left[k_{y}=K_{1}, k_{x}\right]}=\frac{d}{d B}\left[B F_{2}(B)\right]+\zeta_{2} .
$$

(For orbits symmetric about $k_{y}=0, K_{1}=e B D / 2 \hbar c$. For central orbits, $k_{x}=0$.)

Let the maxima, minima, and zero intercepts in $\operatorname{Re}\left[T e^{-i \psi}\right]$ (with the major amplitude factors removed) occur at magnetic fields

$$
B_{0}, B_{1}, \ldots, B_{n}, \ldots \quad\left(B_{n}<B_{m} \text { for } n<m\right) .
$$

At these points, for $i=1,2$,

$$
F_{i}\left(B_{n}\right)=\frac{1}{2} \pi\left(N_{i}-n\right),
$$

where $N_{i}$ is unknown. Then, we define

$$
F_{i}(B)=\frac{1}{2} \pi N_{i}+f_{i}(B) \text {. }
$$

We now know $f_{i}(B)$ at the points $B_{n}$ :

$$
f_{i}\left(B_{n}\right)=-n \frac{1}{2} \pi
$$

and may interpolate in between to obtain $f_{i}(B)$. [It is possible to obtain $f_{i}(B)$ experimentally at points between the $B_{n}$ by using the variable-phase technique discussed in Sec. XI.] Now

$\frac{d}{d B}\left[B F_{i}(B)\right]+\zeta_{i}=\frac{d}{d B}\left[B f_{i}(B)\right]+\zeta_{i}+\frac{1}{2} \pi N_{i}$,

so we know the right-hand side of (9.7) or (9.9) to within the unknown phase constant $\zeta_{i}+\frac{1}{2} \pi N_{i}$.

Further, it may be possible to determine this phase constant to within $\pm \pi$. At the cutoff field $B_{c}$ we know, for symmetric orbits, that $\phi_{D}=\pi=\phi_{s}^{D}$ $-\lambda_{s}^{D}$. Hence,

$$
F_{i}\left(B_{c}\right)=\left(\omega / \omega_{c c}\right) \pi-\zeta_{i},
$$

where $\omega_{c c}$ is the cyclotron frequency at $B_{c}$. Hence, by $(9.10)$ we see

$$
\zeta_{i}+\frac{1}{2} \pi N_{i}=\left(\omega / \omega_{c c}\right) \pi-f_{i}\left(B_{c}\right) .
$$

As the cutoff field can be located fairly accurately from the point of infinite slope in a "extended-zone" plot like Fig. 6, the phase constant $\zeta_{i}+\frac{1}{2} \pi N_{i}$ can be determined with an uncertainty less than $\pi$ in those cases where a well-defined cutoff is observed. Then, combining (9.14), (9.12), and (9.11) with (9.7) or (9.9), as appropriate, the inverse Fermi velocities may be obtained along the path determined by $\left[k_{y}(B), k_{x}(B)\right]$.

For the [100] direction we have obtained these inverse velocities along the path $\left[k_{y}=e B D / 2 \hbar c, k_{x}\right.$ $=0]$ from the experiment, and plotted them in Fig. 14. Here they are compared with the computed inverse APW velocities, as well as those corre- 


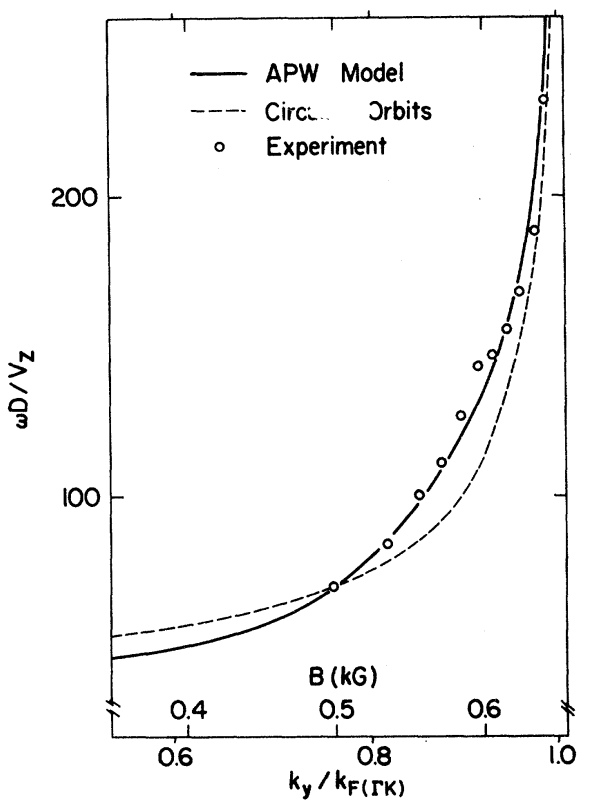

FIG. 14. Velocity component extracted from the transmission spectrum for $\overrightarrow{\mathrm{B}} \|[100]$. $\quad v_{\varepsilon}$ is the velocity component along [011] and $k_{y}$ points along [011] in Fig. 3. $\omega=2 \pi \times 44.8 \times 10^{9} \mathrm{sec}^{-1}, D=235 \mu$.

sponding to circular orbits, to give some indication of the kind of precision obtainable. Referring to Fig. 3 we have thus measured the Fermi-velocity component along [011], point by point, in the angular interval between [011] and [001].

The same measurement made on a sample with its normal in the [010] direction and the magnetic field still in the [100] direction would yield another component of these same velocities. Combining these two results with the symmetry of the orbit would then give all components of the velocities around the entire orbit.

A similar analysis can be performed with the [011] data, but several difficulties make it less valuable. First, the phase constant cannot be determined as precisely, as we do not observe a sharp cutoff field. One must use the point where $v_{y}$ vanishes $\left(\phi_{D}=\frac{1}{2} \pi\right)$, and this cannot be located as accurately. Second, because the stationary point $k_{x}$ depends on $B$, the velocities are determined along a path on the Fermi surface that can only be known after the velocities and cyclotron masses are known. For this reason, these kinds of data are more useful as a very sensitive check of an already existing Fermi-surface model at points away from the symmetry points from which the models are usually determined. In Sec. $\mathrm{X}$ we shall pursue this idea.

\section{COMPARISON OF FERMI-SURFACE MODELS}

The band structures of the noble metals respond sensitively to variations in the crystal potential.
In particular, the position and the width of the $d$ bands depend on the actual choice of potential, whereas Fermi-surface dimensions are somewhat less affected by potential changes. However, the $s-d$ mixing ratio will strongly influence the energy gradients, i.e., the Fermi velocities and cyclotron masses. Since the potential used in the present APW calculation was selected partly because it yields reasonable Fermi-surface parameters and and a correct position of the $d$ bands, ${ }^{4}$ it is not surprising that the calculated velocities differ only slightly from the true quasiparticle velocities. The true velocities can in principle be obtained by fitting a Fourier model as suggested by Roaf ${ }^{13}$ to experimental Fermi-surface dimensions and to cyclotron masses. In order to check the APW results we have therefore calculated the Fermi surface and the velocities using a Fourier model with the expansion coefficients given by Halse. ${ }^{1}$ The two models exhibit only slight differences with respect to dimensions, about $0.5 \% \mathrm{rms}$ over the whole surface. The maximum deviation between the velocities is only $3-4 \%$. (For a more detailed comparison see Ref. 4.)

The $\vec{B} \|[011]$ experiment samples the Fermi surface at general nonsymmetry points, and it is of interest to examine whether the results favor one model over the other despite the small sizes of the differences. In Fig. 15 we have plotted, in analogy to Fig. 9, the zero intercepts of the transmitted spectrum derived from the Fourier model. The results is almost identical to that of the APW model for $B>180 \mathrm{G}$, but in the range $B<180 \mathrm{G}$ there are differences. Using the Fourier model and an over-all phase of $0.6 \pi$, we can now obtain agreement in the entire range $130<B<500 \mathrm{G}$, and the experimental results thus favor the Fourier model over the APW model. One cannot, however, push this too far because of the low-field difficulties associated with the uncertainties in the electric field and the retardation effects as discussed in Sec. VIII B.

\section{SUGGESTED MODIFICATIONS OF THE EXPERIMENT}

The experiment, as described in Sec. IV, measures $(d / d B) \operatorname{Re}\left[T e^{-i \psi}\right]$, where $\psi$ is the phase of the

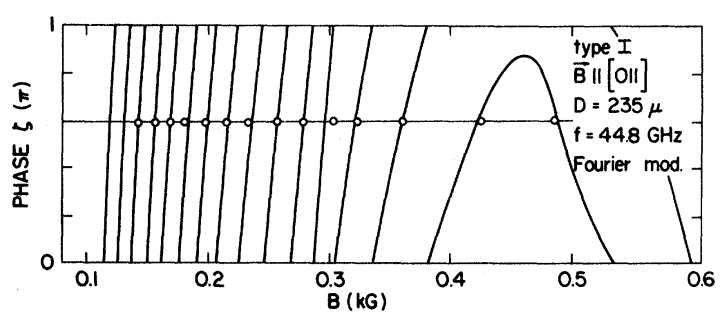

FIG. 15. Zero intercepts of transmitted signal as a function of the over-all phase $\zeta$ (in units of $\pi$ ) for the Fourier model. Best agreement is obtained for $\zeta=0.6 \pi$. 


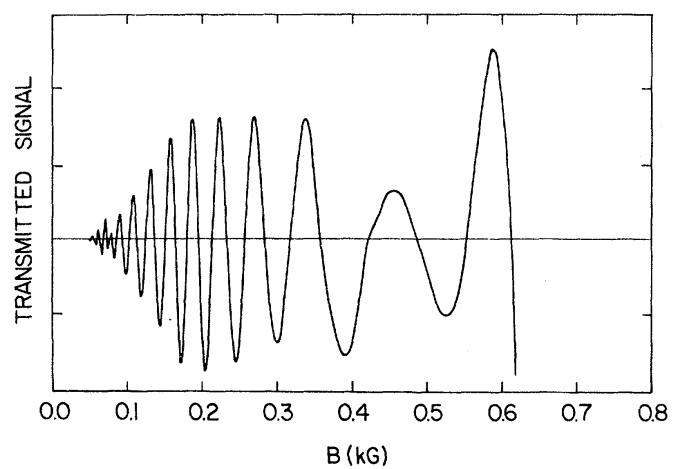

FIG. 16. Transmission spectrum for $\overrightarrow{\mathrm{B}} \|[011]$ with $\overrightarrow{\mathrm{E}} \| \overrightarrow{\mathrm{B}}$, calculated from the APW model.

leak signal. As we have indicated above, it is quite desirable to be able to vary $\psi$. Although the leak phase is not at our disposal, it is possible to effectively vary it by use of an interferometer technique. ${ }^{14}$ Here one lets the microwave generator and local oscillator produce signals which are sent directly into a second i.f. mixer to establish a reference phase. The resultant i.f. signal and the i.f. signal resulting from the path through the sample are fed into a mixer whose dc output is then proportional to

$$
l \cos (\chi-\psi)+\operatorname{Re}\left(T e^{-i \chi}\right),
$$

where the constant phase $\chi$, unlike the leak phase $\psi$, can be altered by varying the phase along the second microwave path.

The virtues of this variable-phase technique are that it aids in identifying the behavior of the phase as a function of magnetic field in plots like Figs. 6 and 10 and offers the possibility of more accurate Fermi-velocity determination by providing values of $f_{i}(B)$ between those of (9.11).

In Sec. IX we mentioned the desirability of removing from the experimental curve the amplitude dependence on $B$. When one is dealing with a magnetic field derivative, this is difficult to do if there are slowly varying base-line signals present. It is therefore better to measure (4.5) or (11.1) directly, without $B$ modulation. This may be done with comparable sensitivity by modulating the amplitude of the input microwave signal to one of the paths, or by modulating the phase $\chi$. In the first case one then measures (11.1) directly, while in the second case one measures the $\chi$ derivative of (11.1) which simply has the effect of shifting $\chi$ by $\frac{1}{2} \pi$.
A modification which may be useful in some cases is to change the direction of the electric field so that $\overrightarrow{\mathrm{E}} \| \overrightarrow{\mathrm{B}}$. This has two effects. First, by replacing $v_{y}$ by $v_{x}$ in (7.26), it removes the zero and phase change in $I_{1}$ that occur when $v_{y}\left(\phi_{D}\right)$ vanishes. This effect is seen by comparing Fig. 16, which shows a calculated transmission spectrum for $\vec{B} \|[011]$ and $\vec{E} \| \vec{B}$, with Fig. 11, where we have $\vec{E} \perp \vec{B}$. Second, it forbids any contribution from belly orbits for which $v_{x}=0$, and so may aid in detecting or sorting out contributions from other parts of the Fermi surface.

Care should be taken to assure that the sample is as close to being plane parallel as possible. If the thickness $D$ is non-uniform, type-II signals can be expected from regions where the thickness is extremal. For type I, however, the trajectories do not hit the second surface at the same $y$ coordinate at which they skimmed the first one. The difference between these $y$ coordinates will increase as $B \rightarrow 0$, and this may cause $D$ in the equations to become, effectively, a function of $B$. If the sample is of uniform thickness but bent, this effect will also occur.

Finally, we should say a word about the experimental parameters used. Generally speaking, higher frequencies will increase the number of oscillations that occur in a given range of $B / B_{c}$. However, for type-I oscillations, higher frequencies will increase the retardation-damping effects, and this may be a controlling factor. Lower frequencies decrease the number of oscillations and make the stationary-phase arguments less valid because of the attendant lowering of the value of $|v|$. If one decreases the sample thickness, the collision damping is decreased, but because $B_{c}$ now occurs at a higher field, the frequency must be increased to maintain the value of $|\nu|$, and then the retardation damping gets worse. Type-II oscillations, of course, are not significantly affected by retardation.

\section{ACKNOWLEDGMENT}

We wish to acknowledge conversations with $\mathrm{J}$. Lebech, J. Mygind, and K. Saermark. The experimental work and most of the calculations were performed in Physics Lab. I, The Technical University of Denmark, and we gratefully acknowledge the kind hospitality of Dr. Saermark there. One of us (J. O. H.) also wishes to thank Dr. J. F. Koch for his hospitality at the University of Maryland where some of this work was completed.
* Supported in part by the National Science Foundation, under Grant No. GP-23571, by the Office of Naval Research, under Grant No. N00014-67-A-0239-0019, and by the Advanced Research Projects Agency, under Grant No. PL-SMOB.
${ }^{1}$ M. R. Halse, Phil. Trans. Roy. Soc. London $\underline{A 265}$ 507 (1969).

${ }^{2}$ R. E. Doezema and J. F. Koch, Phys. Rev. B $\underline{5}$, 3866 (1972).

${ }^{3}$ J. O. Henningsen and D. S. Falk, Phys. Rev. Letters 
26, 1174 (1971).

${ }^{4}$ N. E. Christensen, thesis (Technical University of Denmark, 1970) (unpublished); Phys. Status Solidi $\underline{31}$, 634 (1969).

${ }^{5}$ J. F. Koch and T. K. Wagner, Phys. Rev. 151, 467 (1966).

${ }^{6}$ P. M. Marcus, Natl. Bur. Std. Circ. No. 519 (U. S. GPO, Washington, D. C., 1952), p. 265.

${ }^{7} \mathrm{M}$. C. Jones and $\mathrm{E}$. H. Sondheimer, The Fermi Surface, edited by W. A. Harrison and M. B. Webb (Wiley, New York, 1960), p. 170.

${ }^{8}$ L. E. G. Ah-Sam and M. C. Jones, Alta Frequenza 38, 20 (1969); Phys. Rev. B 1, 3896 (1970).

${ }^{9}$ R. G. Chambers, Proc. Phys. Soc. (London) A65, 458 (1952).
${ }^{10}$ H. D. Drew, Phys. Rev. B $\underline{5}, 360$ (1972).

${ }^{11}$ This field has a larger amplitude a few skin depths below the surface, and in this respect it represents the $B=0$ anomalous skin-effect field better than the expression (7.21).

${ }^{12}$ Our original view that there was no stationary point in this direction does not seem to be correct. We have not included a figure because the relative amplitude and phase of the type-I and type-II signals depend on the details of the electric field. Our model field (7.21) cannot be expected to bring this out correctly.

${ }^{13}$ D. J. Roaf, Phil. Trans. Roy. Soc. London $\underline{\mathrm{A} 255}$ 135 (1962).

${ }^{14}$ J. Mygind, J. Phys. E $\underline{5}, 186$ (1972).

\title{
Size Effect of the Transverse Magnetoresistance in Thin Metallic Films*
}

\author{
Te-chang $\mathrm{Li}^{\dagger}$ and Velio A. Marsocci \\ Department of Electrical Sciences, State University of New York, Stony Brook, New York 11790
} (Received 9 February 1972)

\begin{abstract}
The size effect of the transverse magnetoresistance in thin metallic films has been calculated for the applied magnetic field in an arbitrary direction and with an arbitrary field strength. A new approach using the path-integral formulation has been employed. In calculating the magnetoresistance, it is assumed that the electronic Fermi surface is spherical in momentum space, the electronic scattering at the boundary is completely diffuse, and an isotropic relaxation time exists for the bulk material. In the case where the magnetic field is oriented perpendicular to the surface of the film, the results are identical to the results of Sondheimer's work, which were based on an solution of the Boltzmann equation. The results, for the case with the magnetic field in the plane of the film, are very close to those obtained by Ditlefsen and Lothe; the latter were based on a simulation method. For a low value of the magnetic field, the transverse magnetoresistance has a higher size effect with the field in the plane of the film than for other directions. The theoretical results were found in agreement with the experimental results obtained by Forsvoll and Holwech on the aluminum films.
\end{abstract}

\section{INTRODUCTION}

It is well understood that when the thickness of a metallic thin film is of the same order as, or smaller than, the electronic mean free path, scattering of electrons at the film boundaries become a significant factor, and the effective electrical conductivity is reduced. That is, a geometrical size effect becomes apparent in the conduction for these films. A strict analysis of this problem for the case where only an electric field is applied was first given by Fuchs, ${ }^{1}$ by using a statistical approach to a solution of the Boltzmann equation. In 1950, Sondheimer ${ }^{2}$ and MacDonald and Sarginson, ${ }^{3}$ respectively, obtained solutions for the transverse magnetoresistance in thin metallic films for the cases where the magnetic field is perpendicular to and parallel to the surface of the film. In order to simplify the problem, MacDonald and Sarginson used the bulk value of the Hall field in their cal- culations for thin films. As a consequence their calculations were not consistent with the experimental results. In 1966, Ditlefsen and Lothe ${ }^{4}$ divided the Boltzmann equation into two parts (actually three in their calculation): one which gives the solution for the bulk case and another which gives the solution for the size-effect portion. The latter obtained better results than did MacDonald and Sarginson. It appears that the size effect of the transverse magnetoresistance for the magnetic field in a general direction has not been reported yet.

The Boltzmann differential equation has been extensively used in dealing with the transport processes. Based on the relaxation-time approximation, the Boltzmann equation becomes a linear partial differential equation with known solutions. As is the case for this class of equations the complete solution of the Boltzmann equation is determined by the boundary conditions. There is no 